\title{
Wearables Para Coleta de Dados de Estudantes em Ambiente Escolar: Mapeamento Sistemático
}

\author{
Poliana Nascimento Ferreira $^{1}$, Carla Lopes Rodriguez ${ }^{1}$, Vivian Genaro Motti ${ }^{2}$ \\ ${ }^{1}$ Centro de Matemática, Computação e Cognição, Universidade Federal do ABC Av. \\ dos Estados, 5001 - Bairro S. Terezinha, Santo André, SP, Brazil - CEP: 09210-580 \\ ${ }^{2}$ Department of Information Sciences and Technology, Volgenau School of Engineering \\ - George Mason University - Fairfax - VA, United States - 22030 \\ \{poliana.ferreira, c.rodriguez\}@ufabc.edu.br, vmotti@gmu.edu
}

\begin{abstract}
Wearable devices are used to gather information about users and their environments in an unobtrusive way and are present in several areas, including education. This systematic mapping provides an overview about the current state of research in educational settings where students' data were collected using wearables. 16 articles were selected and analyzed to answer two research questions: how the data has been collected, and the context and goal of the experiments reported. A trend was noticed regarding the usage of inertial commercial sensors involving elementary school children. The most common goal (56\%) concerned offering feedback to the teachers or to the school.
\end{abstract}

Resumo. Dispositivos wearables ou tecnologias vestíveis são utilizados para obter informações sobre o usuário e seu ambiente de maneira não intrusiva e estão presentes em diversas áreas, tais como a educação. Este mapeamento sistemático objetiva traçar um panorama geral sobre o estado atual de pesquisas em salas de aula do ensino básico, que captam dados de alunos com wearables. Foram selecionados 16 artigos sobre o tema para responder duas questões principais de pesquisa: como os dados foram captados e quais o contexto e objetivo dos experimentos. Percebeu-se uma tendência do uso de sensores inerciais comerciais com crianças do ensino fundamental; em relação ao objetivo foi observado que $56 \%$ oferecia feedback para o professor ou para a escola.

\section{Introdução}

Dispositivos wearable, ou vestíveis, são, como o nome diz, tecnologias embutidas em artigos de vestimenta [Motti 2020]. Tecnologias vestíveis tem diversos propósitos de uso, tais como fornecer alertas, facilitar a interação com outras plataformas e dispositivos de maneira simples, mensurar dados do usuário e do ambiente com sensores, ou mesmo apenas para enfeite com fitas de led dinâmicas [Okuno and Guedes 2017, Seymour 2008].

Dentre as possibilidades, destaca-se a coleta de dados com sensores, os quais podem ser classificados de acordo com o tipo de dado a ser coletado. Activity, ou dados de atividade (e.g. acelerômetro, $I M U$ ), Physiological, ou dados fisiológicos (e.g. BPM, $E E G, E D A)$ e Environmental, ou ambientais, que medem dados gerais do ambiente, como luminosidade e umidade, ou aspectos específicos do usuário, como proximidade de outras pessoas ou localização do usuário identificado através de GPS [Motti 2020]. Os sensores estão disponíveis em diversos formatos, e, graças a tal versatilidade, têm sido utilizados em setores como saúde, esportes e educação [Motti 2019], que é o foco deste trabalho. 
A Educação Básica no Brasil é composta por três etapas, a Educação Infantil, o Ensino Fundamental e o Ensino Médio. Segundo a BNCC [Brasil 2018], essas etapas têm por objetivo estimular os alunos no desenvolvimento de competências e habilidades para uma formação humana integral. Nesse sentido, os dispositivos wearable podem auxiliar na ampliação da percepção do professor sobre o aluno, ou turma de alunos, discretamente. Além disso, os dispositivos wearable coletam dados que permitem análises estatísticas para melhorar a aula e ampliar a visão sobre as habilidades motoras e interação entre os estudantes [Watanabe et al.. 2013, Byun et al.. 2018, Saquib et al.. 2018].

O contexto proposto de coleta de dados de alunos por wearables na educação básica, apesar de sua relevância, não tem sido muito explorado como tema de pesquisa. Dessa maneira, é o objetivo deste trabalho desenhar um panorama geral sobre a área, observar tendências e propor caminhos para futuras pesquisas.

\section{Metodologia}

O mapeamento sistemático da literatura é um estudo secundário, que visa a investigação e análise de um tema [Petersen et al.. 2008], nesse caso o uso de wearables para coletar dados de alunos em ambiente escolar. Para esse objetivo, deve-se definir (2.1) questões de pesquisa, (2.2) bases e string de busca, (2.3) critérios de inclusão e exclusão, (2.4) selecionar os trabalhos relevantes ao estudo e, por fim, (3) analisar e apresentar os resultados.

\subsection{Questões de Pesquisa}

Duas questões de pesquisa foram definidas para caracterizar a área selecionada (wearables para coleta de dados em ambiente escolar):

QP1.: Como são coletados os dados através de sensores vestíveis?

A QP1 busca entender quais os sensores utilizados, qual o posicionamento do sensor, como ocorreu o armazenamento dos dados coletados, se o dispositivo é comercial ou customizado para a aplicação, a forma de validação do dispositivo utilizado e metodologia de coleta dos dados (considerando tempo, se ocorreu em contexto naturalístico e como os dispositivos eram colocados e mantidos) mais utilizados para captar os dados.

QP2.: Qual o contexto em que foi aplicado o experimento e seu objetivo?

A QP2 busca entender quais os contextos mais comuns aos quais tem sido dedicados esforços para uso de wearables na educação, considerando-se o objetivo da pesquisa, o público alvo e a(s) métrica(s) para a validação dos dados no contexto proposto.

\subsection{String e Base de Busca}

Para a definição da string de busca, foram selecionados dois contextos principais: relação com educação e uso de sensor vestível. Na Tabela 1 estão representadas as cadeias de pesquisa para bases de dados em inglês e nacionais. A Tabela 2 lista o resultado da busca em cada base, que considerou as palavras no título, resumo ou nas palavras-chave.

Tabela 1. Área relativa e String de Busca

\begin{tabular}{|c|c|c|}
\hline Educação & $\begin{array}{c}\text { "Education" OR } \\
\text { "Classroom" OR "Student" }\end{array}$ & \\
\hline Característica vestível & "Wearable" & "Wearable" OR "Vestível" \\
\hline Ser um sensor & "Sensor" OR "Device" & "Sensor" OR "Dispositivo" \\
\hline
\end{tabular}


Para a seleção dos estudos primários em inglês, foram escolhidas as bases da $A C M$ Digital Library, IEEE Digital Library, ISI Web of Science e Science Direct, por incluírem artigos publicados em diversas conferências, bem como resultados de variadas áreas do conhecimento. Na primeira iteração das buscas, havia também a palavra learning, mas decidiu-se retirá-la para evitar abranger trabalhos sobre "machine learning".

Para os artigos em português, escolheu-se verificar os anais do Simpósio Brasileiro de Informática na Educação - SBIE, do Workshop de Informática da Escola - WIE, dos Workshops do CBIE e as publicações da Revista Brasileira de Informática na Educação. A busca foi feita pelo Google Scholar, que indexa os artigos dos eventos e revistas mencionados (em "exibir artigos publicados em"). É importante observar que, por já tratarem de trabalho em educação, não foi necessária a inclusão da primeira parte da string.

Tabela 2. Resultado da Seleção dos Artigos

\begin{tabular}{|c|c|}
\hline ISI Web of Science & 179 \\
\hline ACM Digital Library & 142 \\
\hline Science Direct & 109 \\
\hline IEEE Digital Library & 97 \\
\hline RBIE, SBIE, WIE, WCBIE & 4 \\
\hline Total de Artigos Selecionados & $\mathbf{5 3 1}$ \\
\hline
\end{tabular}

\subsection{Critérios de Inclusão e Exclusão}

Como critério de inclusão consideraram-se estudos de característica prática que capta dados de estudantes da educação básica em contexto de sala de aula.

Como critérios de exclusão, foram definidos: (1) Ter característica teórica (i.e. não realizar um experimento, ser uma revisão sistemática, entre outros); (2) Não estar em inglês ou português; (3) Não captar dados dos estudantes; (4) O dispositivo utilizado para captação não ser wearable; (5) Não estar relacionado à educação; (6) Ser realizado em ambiente de ensino superior; (7) Ser feito em laboratório ou outros contextos que não a escola; (8) Ter mais de uma publicação (considerou-se a mais recente). Foi definido como critério de qualidade: ser um artigo completo com 5 ou mais páginas.

A classificação para o critério 3 foi determinada seguindo a definição de sensor do tipo de atividade, fisiológicos ou de ambiente (apenas os relacionados à captação de dados do usuário), como definido na Seção 1 . O critério 5 foi usado para eliminar trabalhos que não tinham nenhuma relação com educação: que não mencionavam o público alvo como sendo alunos, e cujo contexto não era a sala de aula (e.x. monitoramento de idosos). Já os critérios 6 e 7 estão relacionados à educação, mas estavam fora do escopo do mapeamento. $\mathrm{O}$ critério 8 foi aplicado apenas na fase de leitura dos artigos completos.

\subsection{Seleção dos Artigos}

A seleção dos artigos se refere à triagem dos 531 artigos retornados pela busca. A primeira etapa consistiu em eliminar os artigos duplicados (22), na segunda etapa foi realizada a leitura do título e resumo para verificar se o trabalho se encaixava em algum dos critérios de exclusão descritos. Após essa leitura, o conjunto de artigos foi reduzido a 44. A terceira etapa incluiu a leitura completa dos artigos que restaram, sendo que 14 atenderam ao critério de inclusão e foram, portanto, selecionados para o mapeamento; mas um deles era repetido, restando 13 artigos. Para esse conjunto de artigos restantes foi utilizada a 
técnica snowball, incluindo a leitura do resumo e depois do texto completo das referências dos artigos selecionados $(\mathrm{n}=576)$. Ao final, contabilizou-se 16 artigos para análise.

A Figura 1a ilustra os anos de publicação e a Figura 1b o país de origem dos artigos analisados. Nota-se que, apesar de não ter sido definido nenhum critério de limite de data, 95\% dos artigos encontrados foram publicados nos últimos 7 anos, indicando que tais estudos têm surgido recentemente em decorrência da evolução e popularização das tecnologias vestíveis. Além disso, é interessante perceber a distribuição dos estudos em relação aos países. Apesar de haver uma concentração maior de trabalhos nos EUA, o que pode se justificar pelas bases de pesquisa e pelo idioma da busca, as demais pesquisas foram realizadas em outros locais como Itália, China, Japão, França, Eslovênia e Reino Unido. Isso indica um interesse pelo tema em cenários e culturas diferentes, mas com predominância de Europa e EUA e limitado envolvimento de países em desenvolvimento.

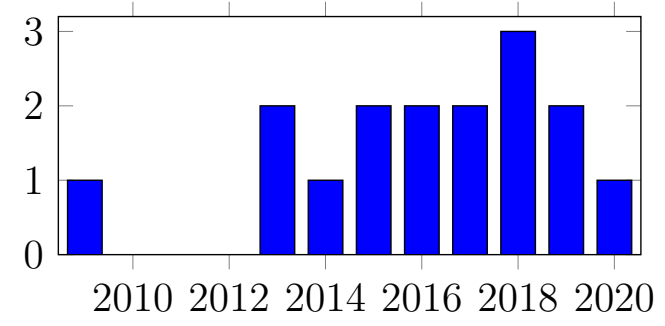

(a) Distribuição dos artigos por ano

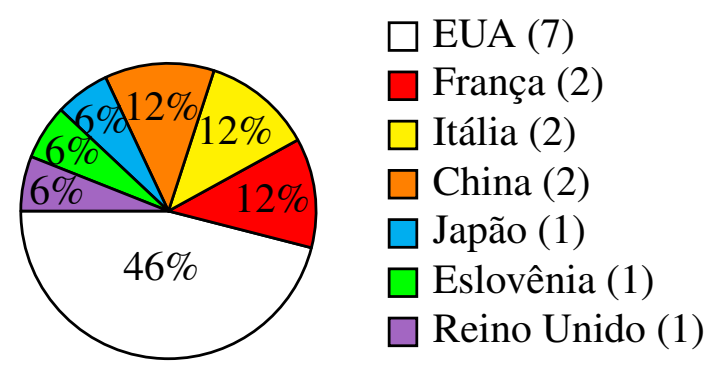

(b) Localização das pesquisas

Figura 1. Informações sobre seleção dos artigos

\section{Resultados e Discussão}

A partir da análise dos 16 artigos selecionados, definiu-se um panorama geral sobre a situação atual da pesquisa em wearables para captação de dados de atividade em contexto educacional. Foram extraídos dados específicos dos experimentos e dispositivos e as duas questões de pesquisa foram respondidas, como descrito a seguir:

QP1.: Como são coletados os dados através de sensores vestíveis?

Como citado na seção 2.1, para responder a essa questão foram extraídos dados relativos a (i) sensores utilizados, (ii) posicionamento no corpo do usuário, (iii) forma de armazenamento dos dados, (iv) se o dispositivo é vendido comercialmente ou customizado, (v) a forma de validação do dispositivo e (vi) metodologia utilizada na coleta.

(i) A distribuição dos sensores está ilustrada na Figura 2. Como 38\% dos estudos utiliza mais de um sensor para captação dos dados, escolheu-se realizar a análise de duas maneiras. A primeira contabiliza todos os sensores usados em cada estudo, assim, os trabalhos com múltiplos sensores são contados uma vez para cada um (n=24) (azul escuro). Já a segunda contabiliza cada trabalho apenas uma vez (azul claro). Analisando os resultados, observa-se uma tendência no uso de sensores de medidas inercial em ambas (42\%/31\%), seguido pelos dispositivos que compõe uma rede de sensores ${ }^{1}$ para posicionamento e identificação de proximidade, como BLE, IR, UWB radio e low-power radio $(25 \% / 19 \%)$, de sensores para captação de informações vitais, como batimento cardíacos,

\footnotetext{
${ }^{1}$ Essa categoria representa dispositivos que se ligam aos demais através de algum protocolo, formando uma rede que mede a proximidade de um aluno (nó) com relação aos demais alunos no grupo
} 
atividade eletrodérmica, temperatura e fluxo de calor (21\%/6\%), câmeras (8\%/6\%), e, por último, com apenas um trabalho, o Global Navigation Satellite System (GNSS) (4\%).

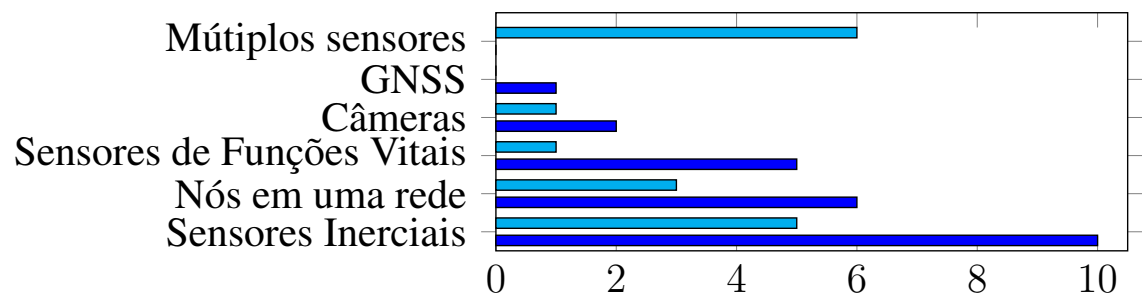

Figura 2. Sensores utilizados

É importante destacar que os sensores inerciais foram usados para diferentes tipos de captura de informação: contar passos, quantificar atividade física, medir as calorias gastas, comparar movimentos entre estudantes, classificar movimentos livres ou verificar gestos. Tal dispositivo é versátil, sendo justificada sua predominância nos experimentos.

(ii) O posicionamento dos sensores deu-se em uma ou mais localizações do corpo. A Figura 3 apresenta as informações referentes às porcentagens de uso em cada local, nota-se que dois locais se sobressaem, punho e peito. Há também uma tendência de posicionamento de sensores inerciais na cintura e de nós em rede no peito.

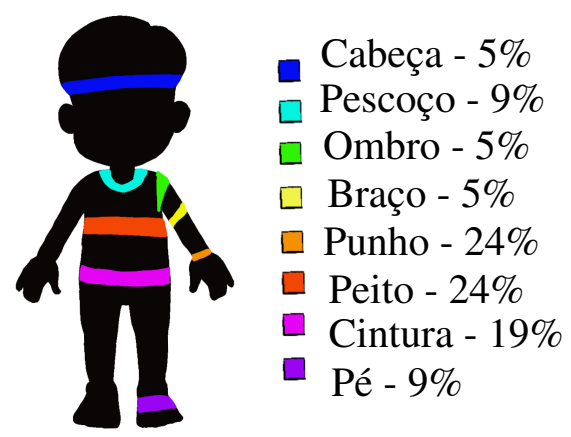

Figura 3. Posicionamento dos sensores

A escolha do posicionamento em relação ao ambiente foi apenas justificada em [Saquib et al.. 2018]. Tal estudo teve a colaboração de professores para a identificação do local menos intrusivo e que pudesse causar menos problemas para os alunos. Os demais trabalhos não incluem a informação ou seguem as diretrizes do fabricante.

(iii) Em relação ao armazenamento, percebeu-se que, em sua na maioria (69\%), o autor optou pela transmissão dos dados para um servidor em tempo real. O restante (31\%) fazia o armazenamento no próprio dispositivo, descarregando os dados para o computador após a coleta. No caso dos dispositivos em rede, foi utilizada a tecnologia sem fio para possibilitar o armazenamento dos dados diretamente em um nó principal da rede, constituindo $100 \%$ de armazenamento no servidor ou computador pessoal em tempo real. Dois trabalhos combinaram os dois modos [Heravi et al.. 2018, Byun et al.. 2018] e dois trabalhos não mencionaram armazenamento [Geršak et al.. 2020, Lee et al.. 2015].

(iv) Observou-se também a tendência do uso de dispositivos comerciais, pela facilidade de manuseio e validação anterior (69\%). Os sensores utilizados incluíam: Fitbit Ultra (2), Fitbit One, Fitbit Flex, ActiGraph GT3X+, G-Sensor-BTS Bioengineering, Hikari Business Microscope, Narrative Clip 2, Opal-APDM Inc., Ubisense RTLS, Empatica E4, GoPro, SocioPatterns, Body-Media Core e "love buckle health" - CoCoQCB2. 
(v) A validação dos sensores diz respeito a verificar se o dado coletado corresponde ao valor esperado quando o sensor é utilizado em um determinado contexto. Três trabalhos não mencionam como validaram, seis realizaram a validação para o público e contexto propostos, utilizando câmeras (3), pelo professor (1), pelos alunos como forma de engajamento (1), pelos pesquisadores com trena e câmera (1) e dois realizaram validação em laboratório. O restante menciona validação externa, seja em outro trabalho ou pela empresa, sendo que apenas um estudo destacou o público específico.

Apenas em um trabalho foi feita uma comparação para selecionar o melhor dispositivo para o objetivo e contexto propostos, comparando as tecnologias para criação da rede de proximidade [Saquib et al.. 2018]. Entretanto, essa comparação pode ter sido feita anteriormente e apenas não descrita no artigo. Além disso, um estudo teve com objetivo a validação específica do sistema no ambiente da sala de aula [Irvin et al.. 2018].

(vi) A parte da metodologia de coleta foi dividida em três pontos: (a) se ela foi feita em uma atividade natural do dia a dia dos alunos ou durante uma atividade planejada, (b) o tempo e (c) a manutenção/posicionamento do dispositivo no aluno.

Apenas três trabalhos (19\%) mencionavam a coleta em uma atividade específica para o experimento, o restante $(81 \%)$ referiu-se a atividades em sala de aula. O tempo total do experimento pode ser visto na Figura 4, sendo que os estudos variaram em coletas em dias específicos, com tempos determinados, até o dia inteiro, todos os dias; a maioria dos estudos foi realizado entre um dia e uma semana. Em relação ao último item, quatro estudos não mencionam como foram realizados e seis indicam que foram operados pelo aluno ou professor, colocando o dispositivo para carregar ao final de certo tempo. No restante, a operação foi feita pelo pesquisador, porém, os autores mencionaram que era interessante desenvolver a aplicação para ser utilizada sem assistência.

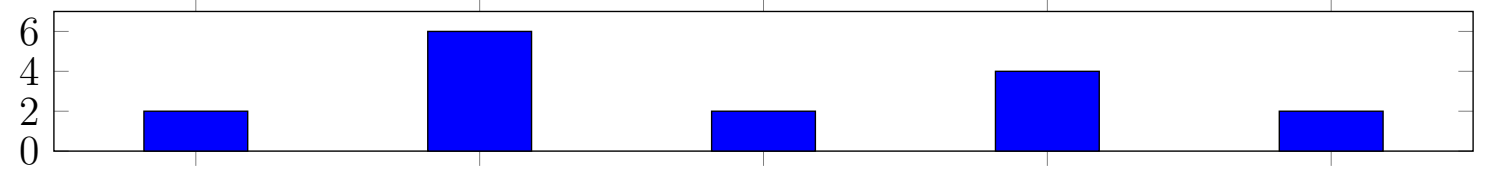

[90 min-1 dia] ]1 dia- 1 sem.] ]1 sem.-1 mês] ]1 mês-3 meses] [12 meses-inf[

\section{Figura 4. Duração da coleta de dados nos experimentos}

Um recurso que chamou atenção em relação a coleta foi a menção ao período de adaptação da criança. No experimento relatado por [Heravi et al.. 2018] houve a participação da criança na decoração do dispositivo, o que foi uma boa estratégia, de acordo com o estudo, para engajar as crianças com o experimento e acostumá-las com o objeto.

Sumário: Os estudos demonstram uma tendência para utilização de sensores inerciais posicionados na cintura, seguidos por sensores para detecção de proximidade entre os alunos, posicionados majoritariamente no peito. Em sua maioria, tais sensores eram comerciais e as informações enviadas em tempo real a um servidor ou computador pessoal. A validação desses dados foi realizada de quatro maneiras, tais como: câmeras para verificação posterior, em laboratório, feita em outro trabalho prévio, ou pelo fabricante do dispositivo. Houve a predominância de estudos que realizaram a coleta em contexto naturalístico no período de um dia a uma semana. Não foi identificada uma tendência para a operação do dispositivo seja essa realizada pelo pesquisador ou pelo professor.

QP2.: Qual o contexto em que foi aplicado o experimento e seu objetivo? 
Como citado na seção 2.1, para responder a essa questão, foram extraídos dados relativos a (i) o objetivo, (ii) o público e (iii) a métrica para a validação no contexto.

(i) Os objetivos dos trabalhos incluíam o estudo da proximidade, interação e localização entre estudantes [Saquib et al.. 2018, Irvin et al.. 2018, Heravi et al.. 2018, Mastrandrea et al.. 2015, Stehlé et al.. 2013, Watanabe et al.. 2013], relação entre sinais fisiológicos e atividade, engajamento e performance acadêmica [Lee et al.. 2019, Geršak et al.. 2020, Miao et al.. 2018, Byun et al.. 2018], identificação de movimentos não usuais em crianças com deficiência [Albinali et al.. 2009], exploração estatística pelos alunos [Lee et al.. 2015, Lee et al.. 2016], identificação dos efeitos de carregar a mochila [Pau et al.. 2015], análise da dieta das crianças na escola e em casa [Zhou et al.. 2019] e identificação de nível de desenvolvimento motor [Sgrò et al.. 2017]. Os estudos foram classificados de acordo com a contribuição: resultados para sala de aula e professor (9) ou relevantes apenas para área de pesquisa (7). Mesmo quando comparados considerando-se o ano de realização, não há uma tendência em relação ao objetivo.

(ii) Em relação ao público, é apresentada na Figura 5a a distribuição de idades, indicando uma predominância de estudos voltados para a idade de 7 a 13, ensino fundamental (69\%), seguido por 0 a 6 anos (19\%) e por 14 a 20 anos (12\%). A quantidade de participantes envolvidos no estudo variou entre 3 e 327 alunos, sendo que dois artigos não mencionaram essa informação. A média foi de 90 pessoas (Figura 5b).

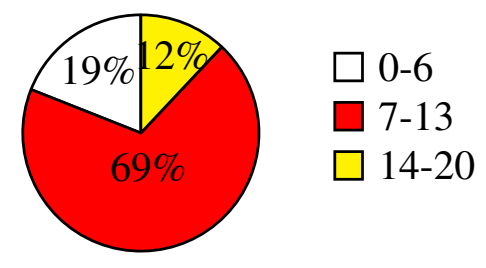

(a) Idade do Público

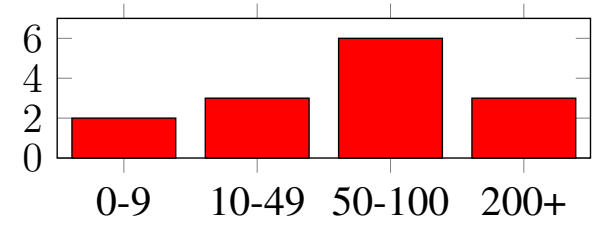

(b) Quantidade dos participantes

\section{Figura 5. Informações sobre público}

(iii) A validação para o contexto inclui pesquisas com os professores, alunos e pais para entender qual foi a perspectiva deles sobre o estudo, qual a viabilidade de uma possível implementação ou qual a influência que os pesquisadores e o dispositivo tiveram dentro do contexto de uso, o que poderia potencialmente ter enviesado os resultados da pesquisa. Além disso, é incluído neste item a visão do próprio pesquisador sobre o que foi realizado, e se ele entende que o resultado fez sentido para o objetivo e contexto.

Apenas cinco estudos apresentaram resultados referentes a opinião do professor ou aluno. Os resultados foram positivos, indicando a aceitação do dispositivo por não ser intrusivo e também por ser, em geral, melhor que outros métodos como de escrever as informações manualmente ou com instalação de câmeras. Os demais estudos reconheceram o impacto da tecnologia em relação ao seu objetivo, mostrando potencial para evolução.

Sumário: A área apresenta uma predominância de estudos com crianças do ensino fundamental, com idade entre 7 e 13 anos, e quantidade de participantes no experimento variando entre 50 e 100 alunos. Além disso, os estudos possuem objetivos variados, sendo tanto voltados para coleta de dados para pesquisa quanto resultados relevantes para o próprio contexto; entretanto, com relação a validação, só foi considerada a perspectiva dos professores em $31 \%$ dos trabalhos. Os pesquisadores reportaram sucesso no cumprimento de seus objetivos, destacando os benefícios do uso dos dispositivos wearables. 
Um aspecto a ser discutido em relação a seleção dos estudo é o fato de nenhum artigo nacional ter sido incluído ${ }^{2}$. Hipóteses foram pensadas como justificativa, levando em consideração a incipiência da exploração de tecnologias móveis na educação no Brasil. Uma delas é a baixa qualidade da infraestrutura para fornecer uma conexão estável, que permita estudos mais específicos como os de coleta de dados por sensores. Outra hipótese é o custo alto e acesso limitado aos componentes no país. Além disso, há uma cultura de uso das tecnologias (de forma geral, mas incluindo as vestíveis) como apoio administrativo e instrumental e não como um recurso de coleta e análise de dados.

Como próximos passos, tem-se melhorar a questão da validação, aumentando a análise da tecnologia a ser empregada e também os testes de uso dentro da sala de aula, a fim de alcançar um dispositivo mais durável, preciso, barato e direcionado ao contexto (e.x. comparação de acelerômetros ou modelos de FitBit). Sugere-se também que as aplicações que visam auxiliar o professor na sala de aula trabalhem a simplificação do uso e manutenção do dispositivo bem como visualização dos dados. Já para as aplicações que visam o pesquisador, sugere-se facilitar o acesso aos dados dos sensores e dos experimentos, os quais não estão detalhados o suficiente para que a pesquisa tenha uma continuidade. Ademais, é importante que o tempo do experimento, assim como o público sejam aumentados [Lee et al. . 2019, Mastrandrea et al.. 2015, Geršak et al. . 2020, Miao et al. . 2018, Stehlé et al.. 2013, Albinali et al.. 2009, Byun et al.. 2018, Watanabe et al.. 2013, Zhou et al.. 2019, Sgrò et al.. 2017].

\section{Considerações finais}

O objetivo deste mapeamento sistemático foi traçar um panorama relacionado ao uso de wearables para coletar dados de alunos em ambiente escolar. A partir da definição da string de busca e questões de pesquisa, foram selecionados e analisados artigos que possuíam característica de aplicação prática, ou seja, que realizaram um experimento no contexto proposto e identificavam o dispositivo utilizado como wearable.

Foi observada uma tendência da coleta de dados utilizando sensores inerciais de atividade e predominantemente comerciais. Além disso, a maioria dos trabalhos realizou o experimento em atividades do dia a dia dos alunos, com uma duração de um a sete dias e envolvendo entre 50 a 100 alunos, 69\% destes no ensino fundamental. Os objetivos de cada artigo variaram, indo desde análise de interação até a identificação dos efeitos de carregar o peso da mochila. Os trabalhos foram classificados a partir de sua contribuição, se apenas para a pesquisa (44\%) ou com feedback para o professor (56\%).

A partir dos resultados encontrados nesse estudo, é possível observar algumas lacunas e fragilidades em relação aos experimentos descritos e a área como um todo, assim como ter um direcionamento para pesquisas futuras. Destaca-se a questão da validação dos sensores especificamente para crianças e para o contexto, trabalhar melhor as informações passadas ao professor e obter a perspectiva deste e dos alunos sobre o proposto. Esses aspectos são importantes tanto para pesquisas voltadas à melhoria do ambiente de sala de aula, por ter um desenvolvimento baseado na visão do público, quanto para as pesquisas voltadas à coleta de dados e análise de comportamento, sendo possível entender as

\footnotetext{
${ }^{2}$ Vista a quantidade reduzida de artigos nas bases nacionais de informática da educação, tentou-se ampliar a string de busca, pesquisando apenas as palavras: "sensor", "dispositivo", "iot", "robótica", dentre outras. Entretanto, os resultados não tratavam do tema proposto.
} 
limitações e influência da presença do pesquisador do ambiente.

Identificou-se, também, algumas limitações deste mapeamento, tais como a escolha das bases de dados, a pesquisa pela string de busca apenas no resumo, a própria string de busca e também possíveis erros humanos na seleção e análise dos artigos. A busca pode ter excluído estudos relevantes por conta do descarte da palavra learning, ou mesmo estudos que não identificaram necessariamente a tecnologia utilizada como wearable, mas que se encaixaria na definição. É importante mencionar que, após a seleção dos artigos, foram encontrados alguns trabalhos como [Schmoelz et al. . 2014] que fazem a mensuração da atividade física no contexto escolar com acelerômetro, entretanto, o sensor não foi caracterizado como wearable, não tendo sido, portanto, encontrado pela busca.

\section{Agradecimentos}

Este trabalho foi realizado com apoio da Coordenação de Aperfeiçoamento de Pessoal de Nível Superior - Brasil (CAPES). Os autores agradecem também à Pró-reitoria de pesquisa e à Pós-graduação em Ciência da Computação da UFABC, pelo apoio concedido.

\section{Referências}

Albinali, F., Goodwin, M. S., and Intille, S. S. (2009). Recognizing stereotypical motor movements in the laboratory and classroom: a case study with children on the autism spectrum. In Proceedings of the 11th international conference on Ubiquitous computing, pages 71-80.

Brasil (2018). BNCC - Base Nacional Comum Curricular. http://basenacionalcomum.mec.gov.br/abase/, acesso em Julho de 2020.

Byun, W., Lau, E. Y., and Brusseau, T. A. (2018). Feasibility and effectiveness of a wearable technology-based physical activity intervention in preschoolers: A pilot study. International journal of environmental research and public health, 15(9):1821.

Geršak, V., Vitulić, H. S., Prosen, S., Starc, G., Humar, I., and Geršak, G. (2020). Use of wearable devices to study activity of children in classroom; case study-learning geometry using movement. Computer Communications, 150:581-588.

Heravi, B. M., Gibson, J. L., Hailes, S., and Skuse, D. (2018). Playground social interaction analysis using bespoke wearable sensors for tracking and motion capture. In Proceedings of the 5th International Conference on Movement and Computing.

Irvin, D. W., Crutchfield, S. A., Greenwood, C. R., Kearns, W. D., and Buzhardt, J. (2018). An automated approach to measuring child movement and location in the early childhood classroom. Behavior research methods, 50(3):890-901.

Lee, V. R., Drake, J. R., Cain, R., and Thayne, J. (2015). Opportunistic uses of the traditional school day through student examination of fitbit activity tracker data. In Proceedings of the 14th International conference on interaction design and children, pages 209-218.

Lee, V. R., Drake, J. R., and Thayne, J. L. (2016). Appropriating quantified self technologies to support elementary statistical teaching and learning. IEEE Transactions on Learning Technologies, 9(4):354-365. 
IX Congresso Brasileiro de Informática na Educação (CBIE 2020)

Anais do XXXI Simpósio Brasileiro de Informática na Educação (SBIE 2020)

Lee, V. R., Fischback, L., and Cain, R. (2019). A wearables-based approach to detect and identify momentary engagement in afterschool makerspace programs. Contemporary Educational Psychology, 59:101789.

Mastrandrea, R., Fournet, J., and Barrat, A. (2015). Contact patterns in a high school: a comparison between data collected using wearable sensors, contact diaries and friendship surveys. PloS one, 10(9):e0136497.

Miao, R., Dong, Q., Weng, W. Y., and Yu, X. Y. (2018). The application model of wearable devices in physical education. In International Conference on Blended Learning, pages 311-322. Springer.

Motti, V. G. (2019). Wearable technologies in education: a design space. In International Conference on Human-Computer Interaction, pages 55-67. Springer.

Motti, V. G. (2020). Introduction to wearable computers. In Wearable Interaction, pages $1-39$. Springer.

Okuno, H. Y. and Guedes, G. P. (2017). Sliclick-em direção à criação de um apresentador de slides vestível. In Anais dos Workshops do Congresso Brasileiro de Informática na Educação, volume 6, page 1280.

Pau, M., Mandaresu, S., Leban, B., and Nussbaum, M. A. (2015). Short-term effects of backpack carriage on plantar pressure and gait in schoolchildren. Journal of Electromyography and Kinesiology, 25(2):406-412.

Petersen, K., Feldt, R., Mujtaba, S., and Mattsson, M. (2008). Systematic mapping studies in software engineering. In 12th International Conference on Evaluation and Assessment in Software Engineering (EASE) 12, pages 1-10.

Saquib, N., Bose, A., George, D., and Kamvar, S. (2018). Sensei: Sensing educational interaction. Proceedings of the ACM on Interactive, Mobile, Wearable and Ubiquitous Technologies, 1(4):1-27.

Schmoelz, C. P. et al. (2014). Padrão de atividade física mensurado por acelerometria no período escolar de crianças do $2^{\circ}$ ao $5^{\circ}$ ano do ensino fundamental.

Seymour, S. (2008). Fashionable technology: The intersection of design, fashion, science, and technology. Springer.

Sgrò, F., Mango, P., Pignato, S., Schembri, R., Licari, D., and Lipoma, M. (2017). Assessing standing long jump developmental levels using an inertial measurement unit. Perceptual and motor skills, 124(1):21-38.

Stehlé, J., Charbonnier, F., Picard, T., Cattuto, C., and Barrat, A. (2013). Gender homophily from spatial behavior in a primary school: A sociometric study. Social Networks, 35(4):604-613.

Watanabe, J.-I., Yano, K., and Matsuda, S. (2013). Relationship between physical behaviors of students and their scholastic performance. In 2013 IEEE 10th International Conference on Ubiquitous Intelligence and Computing and 2013 IEEE 10th International Conference on Autonomic and Trusted Computing, pages 170-177. IEEE.

Zhou, Q., Wang, D., Mhurchu, C. N., Gurrin, C., Zhou, J., Cheng, Y., and Wang, H. (2019). The use of wearable cameras in assessing children's dietary intake and behaviours in china. Appetite, 139:1-7. 